\title{
Covering structure and dynamical structure of a structurally finite entire function
}

\author{
Masahiko Taniguchi, \\ Department of Mathematics, \\ Graduate School of Science, Kyoto University \\ To the memory of Professor Nobuyuki Suita
}

\section{Introduction}

Let $f$ and $g$ be entire functions. We say that $f$ and $g$ determine the same covering structure if they are affine equivalent, i.e. there are similarities $A$ and $B$ such that

$$
f=A \circ g \circ B
$$

and that $f$ and $g$ determine the same dynamical structure if they are affine conjugate, i.e. there is a similarity $A$ such that

$$
f=A \circ g \circ A^{-1} \text {. }
$$

We denote by $\mathcal{C}_{f}$ and $\mathcal{D}_{f}$ the covering structure and the dynamical structure, respectively, induced from $f$. Then, the dynamical structure $\mathcal{D}_{f}$ is smaller than the covering strucure $\mathcal{C}_{f}$ as sets of entire functions. On the other hand, we know the following theorem.

Theorem 1 Suppose that $f$ is a polynomial of degree $N \geq 2$ such that $f^{\prime}$ is not a Ritt polynomial

$$
(z-d)^{m} P\left((z-d)^{\ell}\right)
$$

where $m$ and $\ell$ are non-negative integers, $P$ is a polynomial, $d \in \mathbb{C}$, and $\ell>1$. If another polynomial $g$ satisfies that $g \circ g \in \mathcal{C}_{f \circ f}$, then $g \in \mathcal{D}_{f}$.

This theorem follows from a result by Ritt in [7], or directly from the following simple lemma. 
Lemma 2 (Lenstra-Schneps lemma [8]) Suppose that $P^{(*)}$ and $Q^{(*)}$ are polynomials with $P \circ Q=P^{*} \circ Q^{*}$ and the degrees of $Q$ and $Q^{*}$ are the same. Then there exists a similarity $A$ such that $Q^{*}=A \circ Q$.

For the sake of reader's convenience, we include a proof of Theorem 1.

Proof of Theorem 1. We may assume that

$$
g \circ g=f \circ f \circ C
$$

with a suitable similarity $C$. Then by the Lenstra-Schneps lemma,

$$
g=A \circ f \circ C=f \circ A^{-1} .
$$

Hence letting $D=C \circ A$,

$$
f=A \circ f \circ D \text {. }
$$

Here if $D$ is the identical map, then $A$ is also the identical map, which implies that $f=g$. So suppose that $D$ is not the identical map. Set $D^{\prime}(z)=\delta$ and $A^{\prime}(z)=\alpha$, and we have

$$
\alpha \delta f^{\prime}(D(z))=f^{\prime}(z)
$$

First, if $D$ has a fixed point $w$, then either $\alpha \delta=1$ and $f^{\prime}$ is a non-constant automorphic function with respect to $D$, or $f^{\prime}(w)=0$. In the latter case, suppose that $w$ is a zero of $f^{\prime}$ of order $k$. If $k+1=N$, then $f^{\prime}$ has such a form as $c(z-w)^{N-1}$, which is a Ritt polynomial. If $k+1<N$, then $f^{(k+1)}(w) \neq 0$ and $f^{(k+1)}$ is non-constant. In particular, $\alpha \delta^{k+1}=1$, which implies that

$$
f^{(k+1)}(D(z))=f^{(k+1)}(z),
$$

i.e. $f^{(k+1)}$ is automorphic with respect to $D$. Hence $D$ has a finite order $\ell>1$ and so is $A$.

Thus in any cases, we can find a positive integer $m \leq \ell$ such that

$$
h(z)=(z-w)^{\ell-m} f^{\prime}(z)
$$

is automorphic with respect to $D$, and hence

$$
h(z)=Q\left((z-w)^{\ell}\right)
$$

with a suitable polynomial $Q$. Thus $f^{\prime}$ is a Ritt function.

Finally, suppose that $D$ has no fixed points. Then $\delta=1$ and

$$
\alpha f^{\prime}(D(z))=f^{\prime}(z)
$$


In particular, $\left(f^{\prime \prime} / f^{\prime}\right)(z)$ is a periodic function which is not identically zero. Since $f^{\prime \prime} / f^{\prime}$ is a rational function, it should be a constant, which is impossible.

Remark See [6], where Pilgrim shows that the dynamical structure of an extra-clean Balyi polynomial $P$ is determined by the covering structure of $P \circ P$.

In general, a covering structure $\mathcal{C}_{f}$ corresponds to a complex two-dimensional family consisting of dynamical structures. An exception is the case of a nonlinear polynomial $f$ with a single critical point. When $f(z)=z^{N}$, then $\mathcal{C}_{f}$ contains all

$$
g(z)=c_{1}(z-d)^{N}+c_{2} \quad\left(c_{1} \neq 0\right) .
$$

And for every such $g, \mathcal{D}_{g}=\mathcal{D}_{P_{c}}$ with a suitable $P_{c}(z)=z^{N}+c$. Hence $\mathcal{C}_{f}$ corresponds to a complex one-dimensional family of dynamical structures, i.e.

$$
\left\{\mathcal{D}_{P_{c}} \mid c \in \mathbb{C}\right\}
$$

In this paper, we show a similar theorem as Theorem 1 for the case of structurally finite transcendental entire functions.

The author expresses hearty thanks to Professor Kazuya Tohge for his valuable comments.

\section{The main theorem}

For the definition of structurally finite entire functions, see [9] and [10]. (Also see [5] and [11].) Here we recall the explicit representation and the topological characterization of structurally finite entire functions.

Proposition 3 ([9]) An entire function $f(z)$ is strucuturally finite if and only if

$$
f^{\prime}(z)=P(z) e^{Q(z)}
$$

with suitable polynomials $P(z)$ and $Q(z)$.

Proposition 4 (Cf. [10]) An entire function $f(z)$ is structurally finite if and only if $f$ is a Speiser function and, applying the resolutions of a finite number of singularities of $f^{-1}$ (with respect to a given spider at $\infty$ ) to the covering $f: \mathbb{C} \rightarrow \mathbb{C}$, we have the trivial covering of $\mathbb{C}$ by a countable number of $\mathbb{C}$. 
Here in general, the resolution of a singularity $\sigma$ of $\pi^{-1}$ (which is either a critical point of $\pi$ or a logarithmic singurality of $\pi^{-1}$ ) for a Speiser covering $\pi: R \rightarrow \mathbb{C}$ of $\mathbb{C}$ by a, not necessarily connected, Riemann surface $R$ with respect to a given spider at $\infty$, is the operation defined as follows;

1. cut $R$ along all components of $\pi^{-1}(\ell)$ tending to $\sigma$, where $\ell$ is the leg of the spider ending at the singular value corresponding to $\sigma$, and

2. paste each component of the surface obtained in the first operation along the newly appearing borders over $\ell$, if exist, so that $\pi: R \rightarrow \mathbb{C}$ induces a holomorphic covering $\pi^{\prime}: R^{\prime} \rightarrow \mathbb{C}$ of $\mathbb{C}$ by the resulting, not necessarily connected, Riemann surface $R^{\prime}$.

Theorem 5 Suppose that $f$ is a structurally finite transcendental entire functions such that $f^{\prime}$ is neither a Ritt function

$$
(z-d)^{m} P\left((z-d)^{\ell}\right) e^{Q\left((z-d)^{\ell}\right)}
$$

nor an exponential function

$$
e^{c z+d}
$$

where $P$ and $Q$ are polynomials, $m$ and $\ell$ are non-negative integers, $d \in \mathbb{C}$, $c \in \mathbb{C}-\{0\}$, and $\ell>1$. If another entire function $g$ satisfies that $g \circ g \in \mathcal{C}_{f \circ f}$, then $g \in \mathcal{D}_{f}$.

Theorem 5 is a generalization of Theorem 2 in [12] (cf. [13]). The proof below is different from, and simpler than, that of Theorem 2 in [12]. Also see [1], [2] and [3].

Example 1 Let $f(z)=a e^{b z}+c$ with $a b \neq 0$. Then $\mathcal{C}_{f \circ f}$ contains $g \circ g$ for every $g$ with the same form as that of $f$. Recall that every such $g \in \mathcal{D}_{e_{\lambda}}$, where $e_{\lambda}(z)=e^{\lambda z}$ with a suitable $\lambda \in \mathbb{C}-\{0\}$.

To prove Theorem 5, first we note the following fact, which is an easy consequence of Proposition 4.

Lemma 6 Such a function $g$ as in Theorem 5 is structurally finite.

Proof. Since $f$ is structurally finite, by applying the resolutions of a finite number of suitable singularities of $(f \circ f)^{-1}$, which corresponds to those of $f^{-1}$ for the right $f$ in $f \circ f$, we have a Speiser covering $\pi: R \rightarrow \mathbb{C}$ such that $\pi$ restricted to $\Omega$ is structurally finite for every component $\Omega$ of $R$. We denote by $\mathbf{S}$ the set of all singularities of $(f \circ f)^{-1}$ used to obtain $R$. Let $\mathbf{S}^{\prime}$ be the subset of $\mathbf{S}$ corresponding to singularities of $g^{-1}$ for the right $g$ in $g \circ g$. 
Now $g$ is a Speiser function, for so is $g \circ g=f \circ f$. Suppose that $g$ were structurally infinite. Then by applying the resolutions of all singurarities in $\mathbf{S}^{\prime}$ to $g \circ g: \mathbb{C} \rightarrow \mathbb{C}$, we have a Speiser covering $\pi^{\prime}: R^{\prime} \rightarrow \mathbb{C}$ such that either the number of component of $R^{\prime}$ is infinite or there is a component $\Omega^{\prime}$ of $R^{\prime}$ such that the covering $\left.\pi^{\prime}\right|_{\Omega^{\prime}}: \Omega \rightarrow \mathbb{C}$, i.e. the restriction of $\pi^{\prime}: R^{\prime} \rightarrow \mathbb{C}$ to $\Omega^{\prime}$, has infinitely many singularities of the inverse corresponding to those of $g^{-1}$ for the right $g$ in $g \circ g$.

In the latter case, we can find, either a logarithmic singularity of $\left(\left.\pi^{\prime}\right|_{\Omega^{\prime}}\right)^{-1}$ corresponding to that of $g^{-1}$, or infinitely many critical points $\left.\pi^{\prime}\right|_{\Omega^{\prime}}$ corresponding to those of $g$, for the right $g$ in $g \circ g$. Hence letting $N$ be the number of singularities in $\mathbf{S}-\mathbf{S}^{\prime}$, we can obtain a Speiser covering $\pi^{\prime \prime}: R^{\prime \prime} \rightarrow \mathbb{C}$ by $R^{\prime \prime}$ having more than $N$ components, by applying either the resolution of a logarithmic singularity or the resolutions of a suitable number of critical points such as above.

Thus in any cases, we may assume that $R^{\prime}$ has more than $N$ components, and that the projection $\pi^{\prime}$ restricted to any component of $R^{\prime}$ is structurally infinite. Then, even if we apply resolutions of all the remaining singularities in $\mathbf{S}-\mathbf{S}^{\prime}$ to $\pi^{\prime}: R^{\prime} \rightarrow \mathbb{C}$, we can find a component $\Omega^{\prime}$ of $R^{\prime}$ which is unchanged, and hence $\pi^{\prime}$ restricted to $\Omega^{\prime}$ is structurally infinite. This is a contradiction, which shows the assertion.

Remark Let $\ell$ be an arc either to a critical point of $g$ or to $\infty$. If $\ell$ determines a singularity $\sigma$, then $\ell$ also determines a singulariy $\sigma^{\prime}$ of $(g \circ g)^{-1}$ corresponding to $\sigma$ of $g^{-1}$ for the right $g$ in $g \circ g$.

Also note that, if the singular value $\alpha$ of $g$ corresponding to $\sigma$ is a critical point of $g$, the singularity of $(g \circ g)^{-1}$ corresponding to this critical point of $g$ for the left $g$ in $g \circ g$ also disappears when we apply the resolution of $\sigma^{\prime}$ to $g \circ g: \mathbb{C} \rightarrow \mathbb{C}$.

Thus as in the case of polynomials, Theorem 5 follows from the lemma below, whose proof will be given in the next section, .

Lemma 7 (Transcendental Lenstra-Schneps Lemma) Let $f$ and $g$ be structurally finite transcendental entire functions. Suppose that other structurally finite transcendental entire funcitons $f^{*}$ and $g^{*}$ satisfy the equation

$$
f \circ g=f^{*} \circ g^{*} \text {. }
$$

Then there exists a similarity $A$ such that $g=A \circ g^{*}\left(\right.$ and hence $\left.f=f^{*} \circ A^{-1}\right)$.

Proof of Theorem 5. We may assme that

$$
g \circ g=f \circ f \circ C
$$


with a suitable similarity $C$. Then by Lemmas 6 and 7 , there is a similarity $A$ such that

$$
g=A \circ f \circ C=f \circ A^{-1} .
$$

Hence letting $D=C \circ A$,

$$
f=A \circ f \circ D .
$$

Here if $D$ is the identical map, then $f=g$ as before. So suppose that $D$ is not the identical map. If $D$ has a fixed point $w$, then by Proposition 3 we can conclude as before that $f^{\prime}$ is a Ritt function. If $D$ has no fixed points, $\left(f^{\prime \prime} / f^{\prime}\right)(z)$ is a periodic function not identically zero. On the other hand, $f^{\prime \prime} / f^{\prime}$ is a rational function again by Proposition 3 . Hence it should be a constant, and hence $f^{\prime}$ is an exponential function. Thus we conclude the assertion.

Example 2 If one of $f, f^{*}, g$, and $g^{*}$ is structurally infinite, then the assertion of the above lemma does not necessarily hold. A typical example is a logarithmic lift:

$$
f(z)=e^{z}, \quad f^{*}(z)=z e^{z}, \quad g(z)=z+e^{z}, \quad g^{*}(z)=e^{z} .
$$

Another typical example is

$$
f(z)=e^{z^{2}}, \quad f^{*}(z)=e^{1-z^{2}}, \quad g(z)=\sin z, \quad g^{*}(z)=\cos z .
$$

Here $g$ and $g^{*}$ determine the same covering structure, but the assertion of the lemma does not hold.

On the other hand, we can show the following proposition by the same argument as in the proof of Lemma 6.

Proposition 8 Suppose that $f$ and $g$ are structurally finite, that $g^{*}$ is transcendental, and that $f \circ g=f^{*} \circ g^{*}$ with another entire function $f^{*}$. Then $f^{*}$ is structurally finite.

Finally, professor Masashi Kisaka notified the author the following corollary of the transcendental Lenstra-Schneps Lemma.

Corollary 1 Let $f$ and $g$ be structurally finite transcendental entire functions. Suppose that $f \circ g=g \circ f$. Then $g=A \circ f$ and also $f=g \circ A^{-1}$ with a suitable similarity $A$.

Moreover suppose that neither $f$ nor $g$ has the form

$$
\int_{d}^{z} P\left((t-d)^{\ell}\right) e^{Q\left((t-d)^{\ell}\right)} d t+d
$$

with a suitable integer $\ell>1$, polynomials $P$ and $Q$, and $d \in \mathbb{C}$. Then $f=g$. 
Proof. In this case, $D=A^{-1}$ in the proof of Theorem 5. Hence if $D$ has a fixed point, $f$ should have the form as in Corollary 1. If $D$ has no fixed points, then $f(z)$ should be written as $a e^{b z}+c$, but then $f(z+\beta) \neq f(z)+\beta$ for every $\beta \neq 0$.

Corollary 2 Let $f$ and $g$ be structurally finite transcendental entire functions. Suppose that $f \circ g=g \circ f$. Then the Julia sets of $f$ and $g$ coincide with each other.

Proof. Even if $f \neq g, g=A \circ f=f \circ A$ with a similarity $A$ of a finite order $\ell$. Hence the $\ell$-th iterations of $f$ and $g$ coincide with each other (cf. [4]).

\section{Proof of the transcendental Lenstra-Schneps Lemma}

First by Proposition 3, we can write

$$
\left(f^{(*)}\right)^{\prime}(z)=P^{(*)}(z) \exp \left(Q^{(*)}(z)\right), \quad\left(g^{(*)}\right)^{\prime}(z)=R^{(*)}(z) \exp \left(S^{(*)}(z)\right)
$$

with suitable polynomials $P^{(*)}, Q^{(*)}, R^{(*)}$, and $S^{(*)}$. Here we may assume that $Q^{(*)}(0)=0$ and $S^{(*)}(0)=0$. Let $p^{(*)}, q^{(*)}, r^{(*)}$, and $s^{(*)}$ be the degrees of $P^{(*)}, Q^{(*)}, R^{(*)}$, and $S^{(*)}$, respectively. Then the assumption implies that $q^{(*)}$ and $s^{(*)}$ are positive. Also Proposition 4 gives the following

Lemma $9 q=q^{*}$.

Proof. Apply the resolutions of $s^{*}$ logarithmic singularities of $\pi^{-1}$, corresponding to those of $\left(g^{*}\right)^{-1}$, to the covering

$$
\pi=f \circ g=f^{*} \circ g^{*}: \mathbb{C} \rightarrow \mathbb{C} .
$$

Then the resulting surface $R^{\prime}$ contains infinite number of components $\Omega^{\prime}$ such that each $\Omega^{\prime}$ is biholomorphic to $\mathbb{C}$ and the covering $\left.\pi^{\prime}\right|_{\Omega^{\prime}}: \Omega^{\prime} \rightarrow \mathbb{C}$ induced from $\pi$ has exactly $q^{*}$ logarithmic singularities of the inverse. Further, apply the resolutions of at most $s$ other logarithmic singularities of $\pi^{-1}$, corresponding to those of $g^{-1}$ but not of $\left(g^{*}\right)^{-1}$, to the covering $\pi^{\prime}: R^{\prime} \rightarrow \mathbb{C}$, if exist. Then the resulting surface $R^{\prime \prime}$ also contains infinite number of components $\Omega^{\prime \prime}$, each of which coincides with some component $\Omega^{\prime}$ of $R^{\prime}$, such that the covering $\left.\pi^{\prime \prime}\right|_{\Omega^{\prime \prime}}: \Omega^{\prime \prime} \rightarrow \mathbb{C}$ induced from $\pi^{\prime}$ has exactly $q$ logarithmic singularities of the inverse, which implies that $q=q^{*}$. 
Next, since

$$
f^{\prime}(g(z)) g^{\prime}(z)=\left(f^{*}\right)^{\prime}\left(g^{*}(z)\right)\left(g^{*}\right)^{\prime}(z)
$$

and since the orders of $g$ and $g^{*}$ are finite, we can find a polynomial $T$ such that

$$
Q(g(z))+S(z)-Q^{*}\left(g^{*}(z)\right)-S^{*}(z)=T(z) .
$$

Lemma $10 r=r^{*}, s=s^{*}$, and $b_{s}=b_{s}^{*}$, where we set

$$
S^{(*)}(z)=b_{s^{(*)}}^{(*)} z^{s^{(*)}}+\cdots b_{1}^{(*)} z .
$$

Proof. First recall that $\left|g^{(*)}\right|$ has a growth estimate

$$
\left|g^{(*)}(z)\right|=\left(\gamma^{(*)}+o(1)\right)|z|^{r^{(*)}-s^{(*)}+1} \exp \left(\operatorname{Re} b_{s^{(*)}}^{(*)} z^{s^{(*)}}\right),
$$

with a positive constant $\gamma^{(*)}$ depending only on $g^{(*)}$, as $z \rightarrow \infty$ along a ray in the divergence sectors of $g^{(*)}$ (See for instance, [11] Lemma 4). Here the divergence sectors $\Pi_{j}^{(*)}$ of $g^{(*)}$ is the maximal open set of rays from the origin along which $\left|g^{(*)}\right|$ tends to $+\infty$ :

$$
\Pi_{j}^{(*)}=\left\{\left|\arg z-\frac{-\theta^{(*)}+2 \pi j}{s^{(*)}}\right|<\frac{\pi}{s^{(*)}}\right\} \quad\left(j=0, \cdots, s^{(*)}-1\right),
$$

where we set $\theta^{(*)}=\arg b_{s^{(*)}}^{(*)}$.

Here by the equation (2), the divergence sectors of $g$ and those of $g^{*}$ should be the same, which means that $s=s^{*}$ and $\theta=\theta^{*}$. Then the equation (3) gives that $r=r^{*}$, and $\left|b_{s}\right|=\left|b_{s}^{*}\right|$, which implies the assertion.

Set $Q^{(*)}(z)=a_{q}^{(*)} z^{q}+\cdots a_{1}^{(*)} z$, and take a constant $\alpha$ such that $a_{q}^{*}=\alpha^{q} a_{q}$.

Lemma $11\left|g^{\prime}(z) / \alpha\left(g^{*}\right)^{\prime}(z)\right|$ tends to 1 as $z \rightarrow \infty$ along any ray in the divergence sectors.

Proof. Since $\left|g(z) / \alpha g^{*}(z)\right|$ tends to 1 as $z \rightarrow \infty$ along any ray $\ell$ in the divergence sectors by the equation $(2), \mid Q^{\prime}(g(z)) /\left(Q^{*}\right)^{\prime}\left(\left(g^{*}(z)\right) \mid\right.$ tends to $1 /|\alpha|$ (including the case that $q=1$ ), as $z \rightarrow \infty$ along $\ell$.

Also by differentiating the equation (2), we see that $\mid Q^{\prime}(g(z)) g^{\prime}(z) /\left(\left(Q^{*}\right)^{\prime}\left(\left(g^{*}(z)\right)\left(g^{*}\right)^{\prime}(z)\right) \mid\right.$ tends to 1 as $z \rightarrow \infty$ along $\ell$, which gives the assertion.

Lemma $12 S(z)$ equals to $S^{*}(z)$. 
Proof. By Proposition 3 and Lemma 10, we see that

$$
\frac{\left|g^{\prime}(z)\right|}{\left|\alpha\left(g^{*}\right)^{\prime}(z)\right|} e^{-\left(\operatorname{Re} S(z)-\operatorname{Re} S^{*}(z)\right)}
$$

tends to a non-zero constant as $z \rightarrow \infty$ along any ray in the divergence sectors.

Suppose that there is a $k$ such that $b_{k} \neq b_{k}^{*}$, and let $k_{0}$ be the maximal one among such indice. (Note that $s>k_{0} \geq 1$ by Lemma 10.) Then we can find a ray $\ell$ in the divergence sectors along which

$$
\operatorname{Re}\left(b_{k_{0}}-b_{k_{0}}^{*}\right) z^{k_{0}} \rightarrow+\infty
$$

as $z \rightarrow \infty$. Actually, rays from the origin with angle in suitable $k$ open intervals, the total length of which is $\pi$, in $[0,2 \pi)$ satisfy this condition, and since $k_{0} \neq s$, the set of all such rays can not be disjoint from the divergence sectors. But $\left|g^{\prime}(z) / \alpha\left(g^{*}\right)^{\prime}(z)\right| \rightarrow+\infty$ as $z \rightarrow \infty$ along ever ray in the intersection, which contradicts to Lemma 11.

Finally, by Lemma 12, we can write as

$$
g^{\prime}(z)-\alpha\left(g^{*}\right)^{\prime}(z)=\left(R(z)-\alpha R^{*}(z)\right) e^{S(z)} .
$$

If $R(z)-\alpha R^{*}(z)$ is not identically 0 , then $\left|g(z)-\alpha g^{*}(z)\right|$ grows not slower than $|z|^{-s} \exp (\operatorname{Re} S(z))$ as $z \rightarrow \infty$ along any ray $\ell$ in the divergence sectors. Hence, if $R(z)-\alpha R^{*}(z)$ were not identically 0 for every constant $\alpha$ such that $a_{q}^{*}=\alpha^{q} a_{q}$, then $Q(g(z))-Q^{*}\left(g^{*}(z)\right)$ should grow not slower than $|z|^{-q s} \exp \left(q \operatorname{Re} b_{s} z^{s}\right)$ as $z \rightarrow \infty$ along $\ell$. But this contradicts to the equation (2), which implies that $R(z)-\alpha R^{*}(z)$ is identically 0 for some constant $\alpha$ such that $a_{q}^{*}=\alpha^{q} a_{q}$. Then $g^{\prime}=\alpha\left(g^{*}\right)^{\prime}$, which proves the transcendental Lenstra-Schneps lemma.

\section{References}

[1] I. N. Baker, The iteration of entire transcendental functions and the solution of the functional equation $f\{f(z)\}=F(z)$, Math. Ann. 129, (1955), 174-180.

[2] C. Chuang and C. Yang, Fix-points and factorizationof meromorphic functions, World Scientific 1990.

[3] S. A. Lysenko, On the functional equation $f(p(z))=g(q(z))$, where $p$ and $q$ are "generalized" polynomials and $f$ and $g$ are meromorphic functions, Izvestiya Math., 60, (1996), 963-984. 
[4] S. Morosawa, Y. Nishimura, M. Taniguchi, and T. Ueda, Holomorphic Dynamics, Cambridge Univ. Press, 2000.

[5] Y. Okuyama, Linearization problem on structurally finite entire functions, preprint.

[6] K. Pilgrim, Dessins d'enfants and Hubbard trees, Ann. Scient. Ec. Norm.Sup. 33, (2000), 671-693.

[7] J. F. Ritt, Prime and composite polynomials, Trans. A.M.S. 23, (1922), 51-66.

[8] L. Schneps, Dessins d'enfents on the Riemann surface, L. N. London Math. Soc. 200, (1994), 47-77

[9] M. Taniguchi, Explicit representation of structurally finite entire functions, Proc. Japan Acad. 77 Ser. A. (2001), 68-70.

[10] M. Taniguchi, Synthetic deformation spaces of an entire function, Contemporary Math. 303, (2002), 107-136.

[11] M. Taniguchi, Size of the Julia set of a structurally finite transcendental entire function Math. Proc. Camb. Phil. Soc. 135, (2003), 181-192.

[12] H. Urabe, On entire solutions of the iterative functional equation $g(g(z))=F(z)$, Bull Kyoto Univ. Ed. Ser B. 74, (1989), 13-26.

[13] H. Urabe, Some advances in the theory of factorization of entire or meromorphic functions, Pitman Research Notes in Math. Series 212, (1989), 356-367. 